\title{
A new class of sum rules for products of Bessel functions
}

G. Bevilacqua a) V. Biancalana, and Y. Dancheva

CNISM and Dipartimento di Fisica, Università di Siena, Via Roma 56, 53100 Siena, Italy

T. Mansourb)

Department of Mathematics, University of Haifa, 31905 Haifa, Israel

L. Moi

CNISM and Dipartimento di Fisica, Università di Siena, Via Roma 56, 53100 Siena, Italy

(Dated: May 27, 2022)

We derive a new class of sum rules for products of Bessel functions of the first kind. Using standard algebraic manipulations we extend some of the well known properties of $J_{n}$. Some physical applications of the results are also discussed. A comparison with the Newberger[J. Math. Phys. 23 (1982) 1278] sum rules is performed on a typical example.

PACS numbers: 02.30.Lt, 78.20.Bh

a) Electronic mail: bevilacqua@unisi.it

b)Electronic mail: toufik@math.haifa.ac.il 


\section{INTRODUCTION}

Bessel functions of the first kind $\left(J_{n}\right)$ are among the most ubiquitous special functions of mathematical physics. Their properties are described in several monographs $s^{-\underline{4}} \underline{\underline{4}}$, in the encyclopedic Abramowitz \& Stegun $\underline{\underline{5}}$ as well as in every advanced mathematics textbook.

The generating function identity

$$
\mathrm{e}^{\frac{z}{2}\left(w-\frac{1}{w}\right)} \equiv \sum_{n=-\infty}^{\infty} J_{n}(z) w^{n}
$$

is the key formula used in discussing the frequency modulation (FM) of the electric field of a laser. In fact, a pure sinusoidal FM reads as

$$
\mathbf{E}(t)=\frac{\mathbf{E}_{0}}{2}\left(\mathrm{e}^{i\left(\omega_{L} t+M \sin (\Omega t)\right)}+\text { c.c. }\right),
$$

where $\omega_{L}$ is the laser carrier frequency, $\Omega$ is the modulation frequency, $M$ the modulation index and $M \Omega$ the modulation depth. Using (11) with $w=\mathrm{e}^{i \Omega t}$ one finds the well-known sidebands structure

$$
\mathbf{E}(t)=\frac{\mathbf{E}_{0}}{2} \sum_{n=-\infty}^{\infty} J_{n}(M) \mathrm{e}^{i\left(\omega_{L}+n \Omega\right) t}+\text { c.c. }
$$

In some applications, for instance studying the response of a two-level system to a FM field, one has to deal with expressions like

$$
A_{s}=\sum_{n=-\infty}^{\infty} \frac{J_{n}(M) J_{n-s}(M)}{\gamma+i n \Omega},
$$

where $\gamma$ represents a resonance linewidth. Even if the sum could be done exactly using the Newberger sum rule $e^{\underline{6}}$, the result is quite involved (the details are given in Section IIIA).

Alternatively, in the physical interesting limit $\epsilon=M \Omega / \gamma<1$ (see below for more details), a geometric expansion gives

$$
A_{s}=\frac{1}{\gamma} \sum_{n=-\infty}^{\infty} J_{n}(M) J_{n-s}(M)\left[1-\frac{i \Omega}{\gamma} n-\left(\frac{\Omega}{\gamma}\right)^{2} n^{2}+O\left(\epsilon^{3}\right)\right]
$$

reducing the problem to the evaluation of sums of the form

$$
B_{k, s} \equiv \sum_{n=-\infty}^{+\infty} n^{k} J_{n}(M) J_{n-s}(M)
$$


for integer values of $k \geq 0$ and $s$. To the authors' best knowledge these sums are not addressed in the literature in the general case although special values are known, for instance $B_{0,0}=1$ from 9.1 .76 of [5].

Finding a closed expression for $B_{k, s}$ is the main goal of the present work. The paper is organized as follows. In Section II the main results are derived and discussed, while in Section III some applications are pointed out. Finally Section IV contains the conclusions.

\section{RESULTS}

We start the derivation of the main result by rewriting (1) in a slightly different form

$$
f(y, \theta) \equiv \mathrm{e}^{i y \sin \theta}=\sum_{n=-\infty}^{\infty} J_{n}(y) \mathrm{e}^{i n \theta} .
$$

Deriving the left-hand side $k$ times, one gets

$$
f^{(k)}(y, \theta) \equiv \frac{\partial^{k} f(y, \theta)}{\partial \theta^{k}}=p_{k}(y, \theta) f(y, \theta)
$$

where $p_{k}(y, \theta)$ is a polynomial in $\cos \theta$ and $\sin \theta$ and can be easily recast in the form

$$
p_{k}(y, \theta)=\sum_{n=-\infty}^{\infty} C_{k, n}(y) \mathrm{e}^{i n \theta}
$$

with $C_{k, n}(y)$ polynomials in $y$. An explicit form for these coefficients is worked out in Appendix A. Combining everything one finds

$$
f^{(k)}(y, \theta)=\sum_{n=-\infty}^{\infty}\left(\sum_{q=-\infty}^{\infty} C_{k, q}(y) J_{n-q}(y)\right) \mathrm{e}^{i n \theta}
$$

On the other hand, deriving the right-hand side of (17) one obtains

$$
f^{(k)}(y, \theta)=\sum_{n=-\infty}^{\infty}(i n)^{k} J_{n}(y) \mathrm{e}^{i n \theta} .
$$

An explicit comparison of (11) with (10) is performed in Appendix B. Now consider the

expression $F\left(y_{1}, y_{2}, \theta\right) \equiv f^{(k)}\left(y_{1}, \theta\right) f\left(y_{2}, \theta\right)$. A first form can be obtained using (9) and (7) as follows

$$
\begin{aligned}
F\left(y_{1}, y_{2}, \theta\right) & =p_{k}\left(y_{1}, \theta\right) \mathrm{e}^{i\left(y_{1}+y_{2}\right) \sin \theta} \\
& =\left(\sum_{n=-\infty}^{\infty} C_{k, n}\left(y_{1}\right) \mathrm{e}^{i n \theta}\right)\left(\sum_{m=-\infty}^{\infty} J_{m}\left(y_{1}+y_{2}\right) \mathrm{e}^{i m \theta}\right) \\
& =\sum_{q=-\infty}^{\infty}\left(\sum_{m=-\infty}^{\infty} C_{k, q-m}\left(y_{1}\right) J_{m}\left(y_{1}+y_{2}\right)\right) \mathrm{e}^{i q \theta}
\end{aligned}
$$


In the meantime an equivalent formula can be written using (11)

$$
\begin{aligned}
F\left(y_{1}, y_{2}, \theta\right) & =\left(\sum_{n=-\infty}^{\infty}(i n)^{k} J_{n}\left(y_{1}\right) \mathrm{e}^{i n \theta}\right)\left(\sum_{m=-\infty}^{\infty} J_{m}\left(y_{2}\right) \mathrm{e}^{i m \theta}\right) \\
& =\sum_{q=-\infty}^{\infty}\left(\sum_{n=-\infty}^{\infty}(i n)^{k} J_{n}\left(y_{1}\right) J_{q-n}\left(y_{2}\right)\right) \mathrm{e}^{i q \theta},
\end{aligned}
$$

and, given the completeness of the set $\left\{\mathrm{e}^{i q \theta}, q=0, \pm 1, \pm 2, \ldots\right\}$, the coefficients in both expansions must be equal

$$
\sum_{m=-\infty}^{\infty} C_{k, q-m}\left(y_{1}\right) J_{m}\left(y_{1}+y_{2}\right)=\sum_{n=-\infty}^{\infty}(i n)^{k} J_{n}\left(y_{1}\right) J_{q-n}\left(y_{2}\right) .
$$

This is the central result of the paper and can be considered to be a generalization of the $J_{n}$ addition formula [5, formula 9.1.75], which, in fact, is obtained for $k=0$ noticing that $C_{0, n}=0$ (see Appendix $\mathrm{A}$ ). Besides, with a proper choice of $y_{1}$ and $y_{2}$, different sum rules can be obtained. In fact, substituting $y_{1}=-y_{2}=y$ in (14) and remembering that $J_{n}(0)=0$ one finds

$$
\begin{aligned}
C_{k, q}(y) & =\sum_{n=-\infty}^{\infty}(i n)^{k} J_{n}(y) J_{q-n}(-y) \\
& =\sum_{n=-\infty}^{\infty}(i n)^{k} J_{n}(y)(-1)^{q-n} J_{q-n}(y) \\
& =\sum_{n=-\infty}^{\infty}(i n)^{k} J_{n}(y) J_{n-q}(y) .
\end{aligned}
$$

This gives an answer for the initial problem, namely

$$
B_{k, s}=\frac{1}{i^{k}} C_{k, s}
$$

and the $B_{k, s}$ for the first $k$ values can be read from Appendix $\mathrm{A}$.

The companion case $y_{1}=y_{2}=y$ is worked out with similar algebraic elaborations leading to the formula

$$
\sum_{n=-\infty}^{\infty}(-1)^{n} n^{k} J_{n}(y) J_{n-q}(y)=\frac{(-1)^{q}}{i^{k}} \sum_{m=-\infty}^{\infty} C_{k, q-m}(y) J_{m}(2 y) .
$$

\section{A. Generalizations}

Many generalizations are possible once one dives into the world of generalized Bessel functions $\stackrel{4}{ }$. Let us discuss some cases. 
Consider the function

$$
\mathrm{e}^{i(x \cos \theta+y \sin \theta)} \equiv \sum_{n=-\infty}^{\infty} \tilde{J}_{n}(x, y) \mathrm{e}^{i n \theta}
$$

which arises in the context of general sinusoidal modulation. The $\tilde{J}_{n}(x, y)$ are a kind of "generalized" Bessel functions, which can be given in terms of the usual Bessel functions by means of simple algebraic manipulations (see also equation (11.10) in Dattoli ${ }^{4}$ )

$$
\tilde{J}_{n}(x, y) \equiv \sum_{q=-\infty}^{\infty} i^{q} J_{q}(x) J_{n-q}(y)
$$

The same steps as before lead to the sum rule

$$
2 \sum_{n=-\infty}^{\infty} n \tilde{J}_{n}(x, y)\left[\tilde{J}_{n-q}(x, y)\right]^{*} \equiv(y+i x) \delta_{q, 1}+(y-i x) \delta_{q,-1} .
$$

Furthermore, $k$-order derivatives lead to sum rules with $n^{k}$ which the interested reader can easily work out.

The second generalization is achieved considering

$$
\mathrm{e}^{i\left(y_{1} \sin \theta+y_{2} \sin 2 \theta\right)} \equiv \sum_{n=-\infty}^{\infty} \widehat{J}_{n}\left(y_{1}, y_{2}\right) \mathrm{e}^{i n \theta}
$$

These $\widehat{J}_{n}\left(y_{1}, y_{2}\right)$ "generalized" Bessel functions can be expressed as

$$
\widehat{J}_{n}\left(y_{1}, y_{2}\right) \equiv \sum_{q=-\infty}^{\infty} J_{q}\left(y_{2}\right) J_{n-2 q}\left(y_{1}\right)
$$

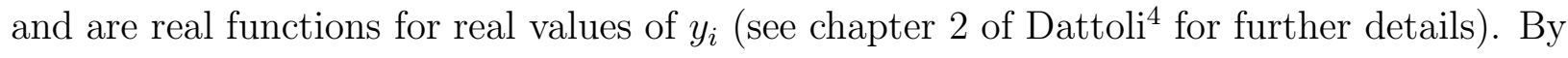
means of the same device one gets

$$
\sum_{n=-\infty}^{\infty} n \widehat{J}_{n}\left(y_{1}, y_{2}\right) \widehat{J}_{n-s}\left(y_{1}, y_{2}\right) \equiv \frac{y_{1}}{2}\left(\delta_{s, 1}+\delta_{s,-1}\right)+y_{2}\left(\delta_{s, 2}+\delta_{s,-2}\right) .
$$

Also in this case higher derivatives lead to other sum rules.

In a complete general setting, one could consider the problem of a FM signal with no special properties, i.e.

$$
\mathrm{e}^{i \phi(t)}=\sum_{n=-\infty}^{\infty} G_{n} \mathrm{e}^{i n \Omega t}
$$

where the only requirement is that $\phi(t)$ is a real and periodic well-behaved function (a more precise statement is given below)

$$
\phi(t)=\sum_{n=-\infty}^{\infty} \phi_{n} \mathrm{e}^{i n \Omega t} .
$$


The $G_{n}$ coefficients are related in a very complicated way to multiple sums of products of ordinary Bessel functions, but the explicit form is not important here. They satisfy the "conservation of energy" sum rule

$$
\sum_{n=-\infty}^{\infty} G_{n} G_{n-s}^{*}=\delta_{s, 0}
$$

Taking the time derivative of (24) and doing the same steps as above we get

$$
\sum_{n=-\infty}^{\infty} n G_{n} G_{n-s}^{*}=i s \phi_{s} .
$$

Equation (27) holds provided that $\dot{\phi}(t)$ can be expanded as a meaningful Fourier series, i.e.

$$
\dot{\phi}(t)=\sum_{n=-\infty}^{\infty} i n \Omega \phi_{n} \mathrm{e}^{i n \Omega t}
$$

must be convergent.

As before, higher order derivatives will give higher order sum rules.

\section{APPLICATIONS}

An example is considered where formula (16) comes in handy. Let us model an optical resonance as a damped harmonic oscillator of unit mass, forced by the electric field of the light beam

$$
\ddot{z}+\gamma \dot{z}+\omega_{0}^{2} z=f \mathrm{e}^{+i \omega t},
$$

where, as usual, the physical oscillator displacement is $x(t)=\Re(z(t))$ and all other symbols have a clear meaning. Such a simple and analytical model avoids all the complications tied to the solution and lets us concentrate on the sum rule. In fact, after the transient, the solution settles into the well-known

$$
z(t)=\frac{f \mathrm{e}^{+i \omega t}}{\omega_{0}^{2}-\omega^{2}+i \gamma \omega},
$$

and the work done by the light field per time unit is

$$
\frac{d W}{d t}=\frac{f^{2}}{4}\left(\mathrm{e}^{i \omega t}+\mathrm{e}^{-i \omega t}\right)\left(\frac{i \omega \mathrm{e}^{i \omega t}}{\omega_{0}^{2}-\omega^{2}+i \gamma \omega}+\frac{-i \omega \mathrm{e}^{-i \omega t}}{\omega_{0}^{2}-\omega^{2}-i \gamma \omega}\right) .
$$

The experimental apparatus usually averages out high frequencies and thus the measured signal is

$$
\left\langle\frac{d W}{d t}\right\rangle=-\frac{f^{2}}{2} \Im\left(\frac{\omega}{\omega_{0}^{2}-\omega^{2}+i \gamma \omega}\right)=\frac{f^{2}}{2} \frac{\omega^{2} \gamma}{\left(\omega^{2}-\omega_{0}^{2}\right)^{2}+\omega^{2} \gamma^{2}},
$$


which, for nearly resonant light $\omega=\omega_{0}+\delta, \delta \ll \omega_{0}$, becomes

$$
\left\langle\frac{d W}{d t}\right\rangle=\frac{f^{2}}{2}\left[\frac{1}{\gamma} \frac{1}{1+\Delta^{2}}+\frac{1}{2} \frac{\Delta^{3}}{\left(1+\Delta^{2}\right)^{2}} \frac{1}{\omega_{0}}+O\left(\frac{1}{\omega_{0}^{2}}\right)\right],
$$

where $\Delta=2 \delta / \gamma$. It is easily seen that the leading term shows the well-known Lorentzian form.

Replacing the forcing term with a frequency-modulated one

$$
\mathrm{e}^{i \omega t} \rightarrow \mathrm{e}^{i(\omega t+M \sin \Omega t)}=\sum_{n=-\infty}^{+\infty} J_{n}(M) \mathrm{e}^{i \omega_{n} t}
$$

on the right-hand side of (29) we get the exact result

$$
\left\langle\frac{d W}{d t}\right\rangle=-\frac{f^{2}}{2} \Im\left[\sum_{s=-\infty}^{\infty}\left(\sum_{n=-\infty}^{\infty} \frac{\omega_{n} J_{n}(M) J_{n-s}(M)}{\omega_{0}^{2}-\omega_{n}^{2}+i \gamma \omega_{n}}\right) \mathrm{e}^{i s \Omega t}\right]
$$

where $\omega_{n}=\omega_{0}+\delta+n \Omega$. To proceed any further it is necessary to simplify the expression $F=\omega_{n} /\left(\omega_{0}^{2}-\omega_{n}^{2}+i \gamma \omega_{n}\right)$. In the optical range it is not restrictive to assume $\omega_{0} \gg \gamma, \delta$ thus getting

$$
F=-\frac{1}{2 \delta+2 n \Omega-i \gamma}\left[1+\frac{(\delta+n \Omega)^{2}}{2 \delta+2 n \Omega-i \gamma} \frac{1}{\omega_{0}}+O\left(\frac{1}{\omega_{0}^{2}}\right)\right] .
$$

Considering only the leading term in $F$ we are faced in (35) with the sums

$$
\frac{1}{i \gamma} \sum_{n=-\infty}^{\infty} \frac{J_{n}(M) J_{n-s}(M)}{1+i \Delta+2 n i \Omega / \gamma}
$$

which are very similar to (44). To proceed any further with the geometric expansion of the denominator, we notice that the product $J_{n}(M) J_{n-s}(M)$ shows the well-known "bridge" structure and it is exponentially small when $|n|>N_{M A X} \approx 2 M$. So if

$$
\left|2 N_{M A X} \Omega / \gamma\right|<1 \Rightarrow\left|\frac{2 N_{M A X} \Omega / \gamma}{1+i \Delta}\right|<1
$$

and the sum can be elaborated as follows

$$
\begin{aligned}
\frac{1}{i \gamma} \sum_{n=-\infty}^{\infty} \frac{J_{n}(M) J_{n-s}(M)}{1+i \Delta+2 n i \Omega / \gamma} & \approx \frac{1}{i \gamma} \sum_{n=-N_{M A X}}^{N_{M A X}} \frac{J_{n}(M) J_{n-s}(M)}{1+i \Delta+2 n i \Omega / \gamma} \\
& =\frac{1}{i \gamma} \sum_{n=-N_{M A X}}^{N_{M A X}} \frac{J_{n}(M) J_{n-s}(M)}{1+i \Delta}\left[1+n \epsilon+n^{2} \epsilon^{2}+O\left(\epsilon^{3}\right)\right] \\
& \approx \frac{1}{i \gamma} \sum_{n=-\infty}^{\infty} \frac{J_{n}(M) J_{n-s}(M)}{1+i \Delta}\left[1+n \epsilon+n^{2} \epsilon^{2}+O\left(\epsilon^{3}\right)\right]
\end{aligned}
$$


where $\epsilon=-i(2 \Omega / \gamma) /(1+i \Delta)$ and the last row is justified because of the exponentially small nature of the terms included.

The inner sum in (35) can now worked out by means of the sum rules developed above, and, after some straightforward algebra, one finds

$$
\begin{aligned}
\left\langle\frac{d W}{d t}\right\rangle=\frac{f^{2}}{2} \frac{1}{\gamma}\{ & \frac{1}{1+\Delta^{2}}+\frac{2 M \Omega}{\gamma} \frac{-2 \Delta}{\left(1+\Delta^{2}\right)^{2}} \cos \Omega t \\
& +\frac{1}{M}\left(\frac{2 M \Omega}{\gamma}\right)^{2} \frac{\Delta\left(\Delta^{2}-3\right)}{\left(1+\Delta^{2}\right)^{3}} \sin \Omega t \\
& \left.+\frac{1}{2}\left(\frac{2 M \Omega}{\gamma}\right)^{2} \frac{3 \Delta^{2}-1}{\left(1+\Delta^{2}\right)^{3}}(1+\cos 2 \Omega t)+\ldots\right\}
\end{aligned}
$$

where the terms in parentheses, besides the d.c. component, represent the higher harmonic modulated absorption. Notice how the $\Delta$ dependence of the $(2 M \Omega / \gamma)^{n}$ term is related to the real and imaginary part of $n$-th derivative of $1 /(1+i \Delta)$. It seems worth stressing that the validity of (41) is limited to modulation depths smaller than the linewidth as pointed out in (38). Usually in typical experimental conditions one is interested in the first harmonic signal, catching it, for instance, by means of a lock-in amplifier. The above formula shows that it is more convenient to use the biggest value of the modulation depth $M \Omega$ compatible with (38).

More generally, using an arbitrary but limited modulation signal as in (25) one finds

$$
\begin{aligned}
\left\langle\frac{d W}{d t}\right\rangle & =\frac{f^{2}}{2} \frac{1}{\gamma}\left\{\frac{1}{1+\Delta^{2}}+\frac{2}{\gamma} \frac{-2 \Delta}{\left(1+\Delta^{2}\right)^{2}} \dot{\phi}(t)+\ldots\right\} \\
& =\frac{f^{2}}{2} \frac{1}{\gamma}\left\{\frac{1}{1+\Delta^{2}}+\frac{2 \sigma}{\gamma} \frac{-2 \Delta}{\left(1+\Delta^{2}\right)^{2}} \frac{\omega(t)}{\sigma}+\ldots\right\}
\end{aligned}
$$

where in the last row the instantaneous frequency $\omega(t)=\dot{\phi}(t)$ is introduced and the "modulation depth" $\sigma=\left|\omega_{\text {MAX }}-\omega_{\min }\right|$ is put in evidence to compare with previous formulae.

\section{A. Comparison with the Newberger sum rule}

As stated in the Introduction, the quantity $A_{s}$ can be summed by means of the Newberger $\underline{\underline{6}}$ sum rule. The result is

$$
A_{s}=\frac{(-1)^{s}}{\gamma} \frac{\pi \gamma / \Omega}{\sinh (\pi \gamma / \Omega)} J_{s-i \gamma / \Omega}(M) J_{i \gamma / \Omega}(M), \quad s \geq 0
$$


where one has to deal with complex-order Bessel functions. Even if this is an exact result, the physics is obscured by the complexity of the formula. For instance, it is not easy to extract the behaviour when $\eta=\Omega / \gamma$ is small. Instead using (5) with $s=1$ we quickly found

$$
A_{1}=-\frac{M}{2 \gamma} i \eta-\frac{M}{2 \gamma} \eta^{2}+\frac{M}{2 \gamma}\left(1+3 M^{2} / 4\right) i \eta^{3}+O\left(\eta^{4}\right)
$$

To proceed further using (42) the product of the Bessel functions must be developed ${ }^{5}$ (formula 9.1.14), and, making use of the properties of the Euler Gamma function ${ }^{5}$ (formula 6.1.31), one gets

$$
A_{s}=\frac{(-1)^{s}}{\gamma}\left(\frac{M}{2}\right)^{s} \sum_{k=0}^{\infty} \frac{\left(-\frac{M^{2}}{4}\right)^{k}(s+2 k) !}{(s+k) ! k !}\left(\prod_{p=1}^{s} \frac{1}{k+p-i \gamma / \Omega} \prod_{p=1}^{k} \frac{1}{p^{2}+(\gamma / \Omega)^{2}}\right)
$$

which, to the authors' best knowledge, is an original elaboration. This formula is still complicated, but better suited to extracting asymptotic behaviour. In fact for $s=1$ we have

$$
\begin{aligned}
A_{1} & =-\frac{M}{2 \gamma}\left(\frac{1}{1-i \gamma / \Omega}-\frac{3 M^{2}}{4} \frac{1}{2-i \gamma / \Omega} \frac{1}{1+(\gamma / \Omega)^{2}}+\ldots\right) \\
& =-\frac{M}{2 \gamma} i \eta-\frac{M}{2 \gamma} \eta^{2}+\frac{M}{2 \gamma}\left(1+3 M^{2} / 4\right) i \eta^{3}+O\left(\eta^{4}\right) .
\end{aligned}
$$

\section{CONCLUSIONS}

We have derived some new classes of sum rules and recursion relations obeyed by Bessel functions of the first kind. The results have broad applications in the physics of modulated excitation for instance in the case of light-matter interaction. A comparison of our results with an elaborated form of the Newberger sum rule is also given.

\section{Appendix A: Determination of $C_{k, n}$}

The starting point is the recursion obeyed by the $p_{k}$ polynomials. In fact, it is easily seen that $p_{0} \equiv 1$, and assuming (요 then follows

$$
p_{k+1}(y, \theta)=\left(\frac{\partial}{\partial \theta}+i y \cos \theta\right) p_{k}(y, \theta)
$$

Projecting this equation as shown in (9) one obtains

$$
C_{k+1, n}=i n C_{k, n}+i \frac{y}{2}\left(C_{k, n+1}+C_{k, n-1}\right) \quad \text { with } \quad C_{0, n}=\delta_{n, 0} .
$$


To the authors' best knowledge this recursion cannot be solved in terms of known special functions, even if the homogeneous right-hand side, namely in $C_{k, n}+i \frac{y}{2}\left(C_{k, n+1}+C_{k, n-1}\right)=$ 0 , is satisfied by the $J_{n}(y)$ itself. The first few loops give

$$
\begin{aligned}
\frac{1}{i} C_{1, n}= & \frac{y}{2}(+1+-1) \\
\frac{1}{i^{2}} C_{2, n}= & \frac{y^{2}}{4}(20++2+-2)+\frac{y}{2}(+1--1) \\
\frac{1}{i^{3}} C_{3, n}= & \frac{y^{3}}{8}(+3+-3)+\frac{3 y^{2}}{4}(+.2--2)+ \\
& \left(\frac{3 y^{3}}{8}+\frac{y}{2}\right)(+.1+-1) \\
\frac{1}{i^{4}} C_{4, n}= & \frac{y^{4}}{16}(+.4+-4)+\frac{3 y^{3}}{4}(+3--3)+ \\
& \left(\frac{y^{4}}{4}+\frac{7 y^{2}}{4}\right)(+2+-2)+ \\
& \left(\frac{3 y^{3}}{4}+\frac{y}{2}\right)(+.1--1)+ \\
& \left(\frac{3 y^{4}}{8}+\frac{y^{2}}{4}\right) 0
\end{aligned}
$$

The recursion is straightforward, but tedious. A simplification is achieved thinking of (A2) as a matrix vector product and implementing it in any computer algebra system.

Alternatively, an approach based on the Faà di Bruno formula can be developed. The Faà di Bruno formula generalizes the chain rule to higher derivatives and can be stated as follows

$$
\begin{aligned}
& \frac{d^{k}}{d x^{k}} f(g(x)) \\
& =\sum_{\left\{m_{j}\right\}} \frac{k !}{m_{1} ! m_{2} ! \cdots m_{k} !} f^{\left(m_{1}+\cdots+m_{k}\right)}(g(x)) \prod_{j=1}^{k}\left(\frac{g^{(j)}(x)}{j !}\right)^{m_{j}} .
\end{aligned}
$$

where the sum is over all $k$-tuples of non-negative integers $m_{1}, \ldots, m_{k}$ satisfying the constraint $1 m_{1}+2 m_{2}+3 m_{3}+\cdots+k m_{k}=k$.

Applying that device to our problem we find

$$
\begin{aligned}
p_{k} & =\mathrm{e}^{-i y \sin \theta} \frac{d^{k}}{d \theta^{k}} \mathrm{e}^{i y \sin \theta} \\
& =\sum_{\left\{m_{j}\right\}} \frac{k !}{\prod_{j} m_{j} ! j !^{m_{j}}}(i y)^{m} \prod_{j=1}^{k}\left(\sin ^{(j)} \theta\right)^{m_{j}},
\end{aligned}
$$


where $m=\sum_{j \geq 1} m_{j}$. Next using $\sin ^{(2 j-1)} \theta=(-1)^{(j-1)} \cos \theta$ and $\sin ^{(2 j)} \theta=(-1)^{(j)} \sin \theta$ we get

$$
p_{k}=\sum_{\left\{m_{j}\right\}} \frac{k !}{\prod_{j} m_{j} ! j !^{m_{j}}}(i y)^{m}(-1)^{\phi} \sin ^{a} \theta \cos ^{b} \theta
$$

where $\phi=\sum_{j \geq 0}\left(m_{2+4 j}+m_{2+4 j+1}\right), a=\sum_{j \geq 1} m_{2 j}$ and $b=\sum_{j \geq 1} m_{2 j-1}=m-a$. Finally expressing the trigonometric functions as

$$
\sin ^{a} \theta \cos ^{b} \theta=\frac{1}{2^{m} i^{a}} \sum_{r=0}^{a} \sum_{r^{\prime}=0}^{b}(-1)^{r}\left(\begin{array}{l}
a \\
r
\end{array}\right)\left(\begin{array}{l}
b \\
r^{\prime}
\end{array}\right) \mathrm{e}^{i\left(m-2 r-2 r^{\prime}\right) \theta}
$$

and substituting in (A5) we find

$$
C_{k, n}=\sum_{\left\{m_{j}\right\}} \frac{k ! i^{b}(-1)^{\phi}(y / 2)^{m}}{\prod_{j} m_{j} ! j !^{m_{j}}} \sum_{r=0}^{a}(-1)^{r}\left(\begin{array}{l}
a \\
r
\end{array}\right)\left(\begin{array}{c}
b \\
\frac{m-n}{2}-r
\end{array}\right)
$$

with $m \geq n \geq-m$, where we follow the convention that the binomial coefficients are zero if the lower index is not an integer or negative or larger than the upper one.

\section{Appendix B: Some new recursion relations}

By comparing the two different forms (10) and (11) of the same quantity, a relation between the coefficients results

$$
q^{k} J_{q}(y) \equiv \sum_{n=-\infty}^{\infty} \frac{1}{i^{k}} C_{k, n}(y) J_{q-n}(y)
$$

The $k=0$ case is the identity $J_{q}(y)=J_{q}(y)$. For $k=1$ we obtain the well-known ${ }^{\underline{5}}$ (formula 9.1.27 first row) three term recursion relation

$$
2 q J_{q}(y)=y\left[J_{q+1}(y)+J_{q-1}(y)\right]
$$

The interesting and, to the authors' best knowledge, new relations are found for $k \geq 2$. In fact for $k=2$ one gets

$$
\begin{aligned}
& \left(\frac{y^{2}}{2}-q^{2}\right) J_{q}(y) \\
& =\frac{y}{2}\left[J_{q+1}(y)-J_{q-1}(y)\right]-\frac{y^{2}}{4}\left[J_{q+2}(y)+J_{q-2}(y)\right] .
\end{aligned}
$$

For higher values of $k$, other relations are easily obtained using the coefficients of Appendix A. 


\section{REFERENCES}

${ }^{1}$ A. Gray and G. B. Mathews, A treatise on Bessel functions and their applications to physics (Macmillan and Co., 1895).

${ }^{2}$ G. N. Watson, A treatise on the theory of Bessel functions (Cambridge University Press, 1922).

${ }^{3}$ B. G. Korenev, Bessel functions and their applications (Taylor and Francis, 2002).

${ }^{4}$ G. Dattoli and A. Torre, Theory and Applications of Generalized Bessel Functions (Aracne Editrice, Rome, 1996).

${ }^{5}$ Milton Abramowitz and Irene A. Stegun, Handbook of Mathematical Functions with Formulas, Graphs, and Mathematical Tables, ninth dover printing, tenth gpo printing ed. (Dover, New York, 1964) ISBN 0-486-61272-4.

${ }^{6}$ Barry S. Newberger, "New sum rule for products of bessel functions with application to plasma physics," J. Math. Phys. 23, 1278 (1982). 